Nesse, R. M. (1984). An evolutionary perspective on psychiatry.

Comprehensive Psychiatry, 25(6), 575-580.

\title{
An Evolutionary Perspective on Psychiatry
}

\author{
Randolph M. Nesse
}

Recent progress in the evolutionary understanding of behavior may greatly assist psychiatry. Although explanations of psychopathology have traditionally emphasized proximate causes of individual differences, consideration of the evolutionary functions of human behavior is essential for psychiatry, just as biology, ethology, and medicine routinely consider both proximate and evolutionary explanations for a variety of phenomena. The methods and data for testing evolu-tionary hypotheses are reviewed, and the ways in which evolutionary principles can help to explain maladaptive behaviors are considered. Some psychiatric symptoms that seem maladaptive may, in fact, serve specific survival functions. Hypotheses are proposed about the possible evolutionary significance of overeating, anorexia nervosa, panic attacks, and sexual disorders, and tests of these hypotheses are considered. By incorporating an evolutionary perspective on psychopathology, psychiatry may share the foundation that evolution provides for the rest of natural science.

TN THE PAST ten years, evolutionary theory has gained substantial new power to explain behaviors and social structures. Unanswered problems from Darwin's time have finally been solved, and a whole class of new hypotheses is being generated and tested. Milestones for this progress include Hamilton's 1968 formulation of the principle of inclusive fitness, and the 1975 publication of Wilson's Sociobiol-ogy. 2-3 The exponential growth since 1975 of publications in this area, attests to the impact of these new ideas. The evolutionary study of behaviors and social structures is quickly becoming an independent discipline. This new power of evolutionary theory to explain human behavior has not been widely appreciated or utilized by psychiatry. Yet, the application of evolutionary principles to psychiatric phenomena makes new questions possible and provides a framework for integrating the seemingly diverse levels of the biopsychosocial model. The territory of evolutionary psychobiology is large, so this presentation must be limited to an outline of the logic of evolutionary explanations in psychiatry, and some examples that illustrate the benefits and problems of testing evolutionary hypotheses about specific psychiatric conditions.

For every biological phenomenon, two different kinds of causes must be understood. Proximate (immediate) causes are those that explain a structure or event in an individual organism. Ultimate (evolutionary) causes are those that explain the existence of a structure or capacity in all members of a species. ${ }^{4}$ Why does the firefly glow? The proximate causes are the environmental precipitants, and the sensory, neural, and chemical mechanisms that result in the flash of light. The question of ultimate cause may be answered only by explaining the evolutionary function of the capacity to glow, and the way that this capacity has increased survival or reproduction in the face of specific forces of natural selection. ${ }^{5}$

Psychiatry has been preoccupied with proximate explanations, and only rarely has considered ultimate explanations. ${ }^{6-9}$ "Why do people experience anxiety?" The

From Randolph M. Nesse,M.D. ofthe Department ofPsychiatry, The University ofMichigan, Ann Arbor, Ml.

Address reprint requests to Randolph M Nesse, MD., Department ofPsychiatry, B 29J7, CFOB, University

of Michigan Hospitals, Box 056, Ann Arbor, Ml 48109.

(a) 1984 by Grune \& Stratton, Inc.

001()..440X/84/2506-0005\$03.00/0 
question is usually answered in terms of the proximate precipitants and mechanisms, physiological and psychological. But we must also understand why the capacity for anxiety evolved in humans. Why do essentially all humans experience jealousy, grief, and loneliness? Both the ultimate and the proximate causes need to be understood for every evolved human capacity.

In biology, ethology, and medicine, evolutionary explanations are not only accepted, they are mandatory. Biologists, too, were once wary of evolutionary explanations. Ultimate causes are now sought even in physiology and molecular biology, where homologous hormones and DNA sequences in vastly different species require evolutionary explanation. 10 Ernst Mayr, in The Growth of Biological Thought, goes so far as to define two biologies: the study of proximate causes is one, the study of ultimate causes is the other. ${ }^{4}$ He emphasizes the legitimacy and importance of both. In Ethology, Hinde states four necessary kinds of questions about the behavior: (1) What is the immediate cause of the behavior? (2) What is the ontogenetic history of the behavior? (3) What is the evolutionary function of the behavior? (4) What is the phylogenetic history of the behavior? ${ }^{11}$ Answers to all four questions are essential to fully explain any behavior, animal or human.

In medicine, considerations of evolutionary function are so taken for granted that they can be hard to recognize. The circulatory system, the immune system, and the digestive system are each defined by their evolved function. The internist recognizes syndromes by the typical pathophysiology which results when the function of a specific system is disrupted. Treatment may be possible on this basis, even if the etiology is unknown. Psychiatry, because it does not recognize the analogous behavioral adaptive systems, is forced to search for either specific etiology or empirical treatment.

The problems encountered in testing hypotheses about the evolutionary function of human traits are real but surmountable. This issue is most comprehensively assessed by Alexander in Darwinism and Human Affairs. ${ }^{\mathfrak{D}}$ The principle that every capacity in every species has an evolutionary function, has some limits. Also, there is no direct fossil record of behavior, and the human species is a special case. But the basic principles of evolution apply equally well to behavioral and emotional capacities in all species. 13 The benefits and problems of evolutionary explanations are best considered using specific examples, but some general issues must first be reviewed.

The logical pattern for an evolutionary analysis of a psychiatric phenomenon is essentially the same as the pattern for analysis of any behavior:

1. Define the phenomenon to be explained.

2 Explicate the proximal cause (environmental, psychological, and neurochemical). 3. Hypothesize possible ultimate causes.

4. Drive predictions from these hypotheses.

5. Test the predictions against the data.

6 If the phenomenon is not an adaptation, but is thought to represent pathology, then find the level, nature, and etiology of the defect in the functional adaptive system affected.

Definition of the phenomenon and explication of the proximate causal chain is the usual pattern of explanations in psychiatry. Environmental precipitants, neurochemical, learning, psychodynamic, and social factors are all important. 
From consideration of these factors, especially the interactions between the environmental situation and the behavior, some clues usually emerge about the evolutionary function of the phenomenon, and a hypothesis may be proposed. Testing hypotheses about evolutionary functions of behavior is essentially the same as for other traits and structures. Evidence of three kinds can be brought to bear: (1) contextual observations, (2) comparative method, and (3) experiment.

Contextual observation is the method of ethology. ${ }^{10 \cdot 14}$ This discipline has so far contributed most of the evolutionary perspective available on psychiatry. ${ }^{15}$. ${ }^{18}$ Evi-dence is provided by environmental and internal states that elicit the behavior, by the details of the behavior itself, and by the effects which result from the behavior. If correct, the hypothesis should be consistent with this evidence, and should predict additional details and correlates. For behavior, the comparative method cannot rely on fossil evidence, but differences between similar behavior patterns in related species should be predicted by the specific ecological adaptations made by each species.

The experimental method can provide evidence when a capacity is disrupted and observations are made of the extent and reason for subsequent decreased fitness. Genetic, surgical, and drug effects can be used, but it is always hard to be sure that the intervention has not accidentally confounded other variables. The first stage of hypothesis testing often involves naturalistic observations which predict the adaptive consequences of depriving an organism of a capacity.

The issue of pathology must be confronted before specific examples are considered. Well and good, one might say, that evolution can provide explanations of normal behavior. But abnormal behavior, by definition, has no adaptive function. How can an evolutionary perspective help us to explain psychopathology? First, it defines normality according to the adaptive consequences of a behavior pattern, without reference to the usual criteria of "freedom from distress," "interference with functioning," or "statistical abnormality." Second, many symptoms can be recognized as distressing, but adaptive. Pain is unpleasant, almost by definition. Yet the early death of people who lack the capacity for pain, makes the adaptive function of this system clear. ${ }^{19}$ Nausea and vomiting are equally aversive, but individuals who did not have these capacities, must often have died of poisoning and infection. Cough is another protective reflex. The very fact that these experiences are so unpleasant may be a result of evolution. These symptoms are often elicited by a threat to homeostasis, yet they are not in themselves pathological, but are normal evolved defenses against pathology. Is anxiety another example? Though unpleasant, it seems to be a universal capacity that is reliably elicited by very specific situations. This suggests that it, too, may be an evolved capacity with an adaptive function. Other human emotional capacities meet the same criteria: loneliness, guilt, grief, jealousy, fatigue, and boredom.

Finally, there are phenomena that are not universal capacities and those that are maladaptive even from an evolutionary perspective. One approach has been to ignore this distinction and insist that there is a function for everything. But, to blithely assume adaptive functions for schizophrenia, endogenous depression, suicide, and child abuse, makes a mockery of the evolutionary perspective. Proposed functions must be justified in terms of their impact on genetic fitness. Just because we all have the capacity for psychosis, does not make it adaptive. We all have the 
capacity for a positive Babinski sign, but this is a release phenomenon, not an evolutionary adaptation. For a pathological symptom or syndrome it is necessary to: (1) Identify the adaptive system involved; (2) Explicate the proximate mechanism that normally operates the system; (3) Identify the locus, nature, and etiology of the specific defect in that system; and (4) Assess the effect of that defect on the adaptive function of the system. In particular, disruptions of a control system need to be distinguished from more fundamental disruptions of the capacity itself. In most mental illness, normal human emotions and behavior patterns are elicited too early or late, too intensely or mildly, or in response to the wrong stimulus. In others, the capacity is absent, or the response is fundamentally deformed.

It may be helpful to emphasize what is not being argued. It is not proposed that genetic differences explain an individual's behavior. And it is not proposed that all behavior is innate. The capacity for individual learning is present in so many species precisely because it affords such a substantial selective advantage. The thesis is, instead, that almost every behavior and emotional capacity and tendency shared by all humans, is a result of evolution, and therefore serves an adaptive function that increases the survival and reproduction of that individual's genes.

Eating disorders are among the most common, most treated, and most studied of psychiatric problems, yet the adaptive functions of eating and satiety, and the evolution of the responsible mechanisms, are rarely considered. What selective pressures shaped the mechanism that controls the quantity of food consumed? There are two motivating forces, hunger and satiety, each with specific evolved anatomic localization and neurohumoral control. For hunger, the selective forces are clear. An organism that does not eat, quickly dies, and its genes are eliminated from the pool. Furthermore, periods of limited food supply have selected for the capacity to store fat, and the behavioral tendency to eat enough to fill these stores when necessary. Pregnancy is one condition in which additional fat stores are particularly adaptive. Females with the capacity and tendency to store additional fat during pregnancy, are able to nurse their infants through a famine; the infants of mothers who do not gain weight often die. The clinical observation that obesity often begins with pregnancy or the administration of birth control pills supports this hypothesis. Detailed analysis of the responsible mechanism should be possible.

But how could the satiety mechanism augment survival? Large individuals need more food to sustain themselves, and will therefore provide less for their mates and offspring. They will also be slower and therefore less effective as hunters and more vulnerable as prey.

Why are there more overweight than underweight people in the industrial societies? Proximate explanations look at the food types and availability, and at energy expenditure, but the ultimate explanation is that some natural selection forces have shaped a hunger system that absolutely ensures adequate food intake, while other selection forces have shaped a satiety mechanism that is feeble by comparison.

Anorexia nervosa is more clearly pathological, yet the remarkable consistency of this syndrome suggests the presence of a possible adaptive mechanism. Patients usually describe a period of strict dieting, followed by an overwhelming fear of obesity that is associated with preoccupation with food and fear of gorging or actual episodes of bulimia. 20 A speculative hypothesis emerges. 
Does there exist a mechanism, evolved after millions of years of feast and famine, that prepares individuals for periods of food shortage, by inducing eating behavior to store calories? Diet-induced hypoglycemia could elicit this behavior pattern and result in impulses to gorge. Women with a socially induced fear of obesity and a strong ability to resist impulses, might try to control their weight by more strict dieting, thus setting up a positive feedback cycle that would make things still worse. This has obvious implications for obesity. Can dieting cause obesity? Perhaps. This hypothesis gives rise to the nonobvious testable prediction that, in many species, individuals exposed to periods of food deprivation should gain more weight than otherwise identical individuals given continuous access to food. The hypothesis seems less farfetched when one considers the many species that respond to envi-ronmental cues anticipating the hibernation season by gaining large amounts of weight.

Panic disorder is another very consistent syndrome. ${ }^{21}$ Patients with panic disorder report attacks coming "out of the blue," that begin with overwhelming terror and perhaps a moment of immobility. In seconds, patients experience palpitations, shortness of breath, sweating, an empty feeling in the stomach, and a wish to run home to be with trusted relatives. Repeated attacks lead to agoraphobia, as patients avoid locations where panic attacks have occurred. Agoraphobics also typically avoid certain other situations, including wide open spaces, being alone, being far from home, and being in any situation in which they might be, or feel, trapped.

Cannon's "fight or flight" response is an almost perfect description of the signs and symptoms of a panic attack. 22 The rapid heart rate, pale skin as blood is shunted to muscle, the cooling sweat, the hyperventilation to oxygenate the blood-all are adaptive preparations for fight or flight. But fight with or flight from what? From predators and hostile humans. Not only are the characteristics of the reflex consistent with this, but the environmental precipitants are too. Of course the organism is made apprehensive by wide open spaces without trees, and by locations in which it may be trapped, so are most prey animals. The momentary immobility at the onset of danger is a camouflage device used by mice and rabbits as well as primates. Why flee home and seek out trusted relatives? Every social animal does the same. Why are women more susceptible? Probably because they are usually more vulnerable. Why should severe apprehension result from one-time learning? Individuals who had no fear when they saw a lion for the second time on the open plain did not survive.

As psychophysiological capacities intimately related to reproduction, sexual responses are particularly susceptible to evolutionary analysis. Every book on sexual disorders contains extensive discussion about the causes and treatment of premature ejaculation in men and delayed orgasm in women. By why do men usually reach orgasm more rapidly than women? Well, what if it were reversed? A woman who consistently reached orgasm quickly and stopped coitus before her mate reached orgasm would rarely reproduce. A man whose orgasm was consistently delayed would also be at a reproductive disadvantage. Genes that cause such tendencies are quickly eliminated. Natural selection did not shape us for harmony and pleasure, but, inevitably, for effective reproduction. That anxiety causes impotence is common knowledge. The reason may be that copulating couples who continued when danger appeared, had even shorter lives than their contemporaries. 
These examples are few and incomplete. The specific predictions that offer tests for each hypothesis deserve more detailed discussion, and additional examples could consider the evolutionary significance of other psychiatric symptoms, the emotions, the personality types, and the mental structures. But here the goal must be simply to demonstrate the legitimacy and importance of seeking ultimate explanations and the ability of evolutionary theory to help us find these explanations. At this point, evolutionary psychobiology offers many new questions and few firm answers. But by recognizing the evolutionary origins of human nature, and by considering the adaptive significance of every human capacity, psychiatry may gradually come to share the firm foundation that evolution provides for all of natural science.

\section{REFERENCES}

1. Barash DP: Sociobiology and Behavior. New York, Elsevier, 1977

2 Hamilton WD. The genetical theory of social behavior. J Theor Biol 7:1-52, 1964

3 Wilson EO: Sociobiology. Cambridge, MA, Harvard Univ Press, 1975

4 Mayr E: The Growth of Biological Thought. Cambridge, MA, Belkamp Press, 1982

5 Lloyd JE: Mimicry in the sexual signals of fireflies. Sci Am 245:138-147, 1981

6 McGuire MT: Sociobiology: Its Potential Contributions to Psychiatry. Perspect BiolMedpp50-69,Autumn, 1979

7. McGuire MT, Essock-Vitale SM: Psychiatric disorders in the context of evolutionary biology: A functional classification of behavior. J Nerv Ment Dis 169:672-686, 1981

8 Alexander RD, Tinker DW (eds): Natural Selection and Social Behavior. RecentResearch and New Theory. New York; Chiron Press, 1981

9. Chagnon NA, Irons W (eds): Evolutionary Biology and Human Social Behavior: AnAnthropological Perspective. North Scituate, MA, Duxbury Press, 1979

10. Roth J, LeRoith D, Shiloach J, et al: The evolutionary origins of homones, neuro-transmitters and other chemical messengers. New Engl J Med 306:523-527, 1982

11. Hinde RA: Ethology: Its Nature and Relations with Other Sciences. New York, Oxford, 1982

12 Alexander RD: Darwinism and Human Affairs. Seattle, Univ Washington Press, 1979

13. Wilson EO: On Human Nature. Cambridge, MA, Harvard Univ Press, 1978

14. Lorenz KZ: The Foundations of Ethology. New York, Springer-Verlag, 1981

15. McGuire MT, Fairbanks LA (eds): Ethological Psychiatry. Gune \& Stratton, New York, 1977

16 White NF (ed): Ethology and Psychiatry. Toronto, Univ Toronto Press, 1974

17. Polsky R, McGuire MT: An ethological analysis of manic-depressive disorder. J MentDis 167:56-65, 1981

18 Hinde RA: Towards Understanding Relationships. New York, Academic Press, 1979

19. Stemback RA: Congenital insensivity to pain: A critique. Psycho! Bull 60:254-264, 1963

20. Buch H: Eating Disorders. New York, Basic Books, 1973

21. Klein DF: Anxiety reconceptualized, in Klein DF, Rabkin JG (eds): Anxiety: New ResearchandChanging Concepts. New York, Raven Press, pp. 235-264, 1981

22 Cannon WB: Bodily Changes in Pain, Hunger, Fear and Rage. New York, Appleton, 1915 